\title{
Análisis de Tráfico para un Servicio de Vídeo bajo Demanda sobre Redes HFC usando el Protocolo RTMP
}

\author{
Wilmar Y. Campo ${ }^{(1)}$, Jose L. Arciniegas* ${ }^{(1)}$, Roberto García ${ }^{(2)}$ y David Melendi ${ }^{(2)}$ \\ (1) Universidad del Cauca, Facultad de Ingeniería Electrónica y Telecomunicaciones, Depto. de \\ Telemática, Calle 5 No. 4-70, Popayán-Colombia (e-mail: wilicampo@unicauca.edu.co; \\ jlarci@unicauca.edu.co) \\ (2) Universidad de Oviedo, Depto. de Informática, Campus de Viesques s/n, 33204, Gijón-España \\ (e-mail: garciaroberto@uniovi.es; melendi@uniovi.es)
}

* Autor a quien se debe dirigir la correspondencia

Recibido Dic. 30, 2009; Aceptado Feb. 22, 2010; Versión Final recibida Abr. 22, 2010

\section{Resumen}

En este artículo se presenta el análisis, caracterización estadística y modelado de un servicio de vídeo bajo demanda en una red de cable HFC (Híbrida de Fibra y Coaxial). Los servicios de audio y vídeo producen un elevado consumo de recursos en las redes de comunicaciones. Por ello, se hace necesario llevar a cabo estudios detallados que permitan evaluar el impacto de la implantación de estos servicios y su efecto sobre otras aplicaciones que se estén ejecutando en la misma red. El servicio de audio y vídeo estudiado utiliza el protocolo RTMP para la transmisión de tráfico multimedia entre los clientes y el servidor. Con el objetivo de evaluar el rendimiento del servicio en las redes de cable, se han analizado varios escenarios con diferentes configuraciones del protocolo de control de acceso al medio en las redes HFC.

Palabras claves: análisis de tráfico, modelado, vídeo bajo demanda, red de cable, RTMP.

\section{Traffic analysis for video on demand services over HFC networks using RTMP protocol}

\begin{abstract}
In this paper, the analysis, statistical characterization and modeling of a video on demand (VoD) service running on a cable network HFC (Hybrid Fiber Coaxial) is presented. Audio and video services produce a high consumption of resources on the network infrastructure. Thus, it is necessary to perform detailed studies that allow evaluating the impact of implementing these services and their effect on other services running on the same network. The audio and video service uses the RTMP protocol for multimedia traffic transmission between clients and server. With the objective of evaluating the service performance on cable network, several scenarios with different configurations of the media access control protocol in HFC networks have been analyzed.
\end{abstract}

Keywords: traffic analysis, modeling, video on demand, cable networks, RTMP. 


\section{INTRODUCCIÓN}

Los proveedores de servicios de Internet han incrementando continuamente las velocidades de acceso que proporcionan a sus clientes. Gracias a estas mejoras en el ancho de banda, han aparecido con gran fuerza servicios basados en la transmisión de tráfico de audio y vídeo denominada flujo multimedia (del inglés streaming). Debido a esta tecnología, el uso de servicios de Vídeo bajo Demanda (VoD, por sus siglas en inglés) es cada vez más frecuente en Internet, cientos de portales ofrecen servicios específicos, tanto sociales como comerciales de VoD. Por ello, la caracterización del tráfico generado por los servicios de flujo multimedia, juega un papel muy importante tanto para evaluar el rendimiento de los nuevos servicios de audio y vídeo, así como para la implicación que puedan tener sobre el resto de aplicaciones en la red.

El servicio de $\mathrm{VoD}$, que es provisto por un servidor, consiste en una aplicación que espera, procesa y sirve peticiones de uno, o varios clientes. La petición, contiene un comando donde el cliente solicita el vídeo que desea recibir. Una vez el servidor ha recibido el comando de reproducción, empieza a transmitir el vídeo. Estos datos, al llegar a la aplicación cliente se almacenan temporalmente para absorver posibles cortes de la reproducción debidos a la latencia y la variación de la señal en tiempo (jitter). En los casos que se vacía el almacenamiento temporal se producen bloqueos (cortes). Otro efecto son las distorsiones que se producen al perderse algunos fragmentos del flujo de datos que envía el servidor. El flujo multimedia es una técnica que permite reproducir el vídeo a la vez que se va recibiendo en el cliente.

El flujo multimedia antes de ser transmitido debe ser codificado, con el objetivo de reducir al mínimo tanto el espacio, como el ancho de banda. La calidad óptima de servicio para el VoD, y por extensión para cualquier clase de servicio remoto, es aquella en la que el usuario no es capaz de determinar si el vídeo se está re-transmitiendo desde un punto lejano de la red o está almacenado en su propio computador (Melendi et al., 2003).

Por otro lado, las redes HFC son el resultado de un proceso evolutivo que se ha llevado a cabo sobre las redes de distribución de televisión por cable. La transformación de las redes de cable se debe al aumento en la oferta de canales como estrategia de la expansión del negocio, lo que implica una demanda de ancho de banda. Esto ha impulsado a mejorar la tecnología sobre las que estaban implementadas. Pasando así, de la utilización de cables coaxiales, a la utilización de tecnología de transmisión óptica que ofrece grandes posibilidades en fiabilidad y capacidad de transmisión, dando origen a la redes HFC multiservicio en las que es posible la creación de topologías de red susceptibles de transportar señales bidireccionales (Álvarez-Campana et al., 2009; España, 2003; ETSI EN 300 744, 2004; CableLabs, 2006). Sin embargo, el crecimiento experimentado en el volumen de tráfico y del número de usuarios y aplicaciones, implica un aumento del consumo de recursos tanto de la red como de sus dispositivos asociados. Esta situación puede conducir a una degradación del rendimiento de las redes HFC.

Es por ello que la realización de análisis de tráfico previos, pueden ayudar a determinar el impacto de la implantación de nuevos servicios y la previsión oportuna de situaciones no deseadas. En esta línea, los modelos de tráfico son esenciales para la evaluación de las prestaciones de las redes de telecomunicaciones.

En este artículo se presenta el análisis de tráfico en un contexto real de una red HFC, mediante la monitorización de un servicio de VoD basado en tecnología Flash debido a su gran popularidad en el mercado. Asimismo, se analiza el comportamiento del protocolo RTMP (Real Time Messaging Protocol) y se caracteriza estadísticamente el tráfico tanto en los clientes como en el servidor de VoD. Esta caracterización ha permitido diseñar un modelo del servicio que ha sido validado y probado en diferentes escenarios de redes de cable utilizando el protocolo de control de acceso al medio DOCSIS (Data Over Cable Service Interface Specification) en la red HFC.

Para su descripción, el artículo está organizado en las siguientes secciones: trabajos relacionados, entorno de experimentación, análisis de tráfico, modelo del servicio de flujo multimedia, resultados y finalmente las conclusiones. 


\section{TRABAJOS RELACIONADOS}

El uso de servicios de VoD cada vez es más frecuente en Internet. Así los servicios de VoD y su implementación han aportado nuevos retos a la comunidad científica. El diseño de estos sistemas involucra diferentes áreas como sistemas de tiempo real, sistemas de archivos de altas prestaciones, calidad de servicio, protocolos de comunicaciones, formatos de compresión, criptografía, sistemas de procesamiento, etc.

Existen trabajos relacionados como (Melendi et al., 2006) el cual presenta un estudio estadístico tanto del comportamiento de los usuarios como del tráfico generado por el flujo multimedia, teniendo en cuenta el protocolo RTSP (Real Time Streaming Protocol), el cual se encuentra claramente especificado en el RFC 2326. Sus resultados les permiten desarrollar un modelo de simulación. En (Merwe et al., 2002) se estudia el tráfico asociado con dos de las mayores categorías de contenidos de flujo multimedia: la primera, sobre demanda cuyo contenido es pregrabado y la segunda, la difusión en vivo, en donde se usan los registros de un servicio comercial para analizar un gran número magnitudes tales como caracterización del servicio, popularidad, carga de la red, etc., sin embargo dicho estudio no se hace sobre la red HFC. En (Loguinov y Radha, 2001) se realiza el análisis de flujo multimedia para clientes de línea conmutada para vídeos codificados mediante MPEG-4 y se analizan parámetros de red como paquetes perdidos, retardos y variación de la señal en el tiempo. En (Mena y Heidemann, 2000) se presentan los resultados de un breve estudio para examinar el tráfico generado desde un servicio de audio en Internet usando el formato RealAudio, los resultados muestran que los flujos de audio exhiben una consistencia en cuanto a la tasa de datos y son considerablemente más persistentes que las conexiones HTTP. En (Chen et al, 2003), se centran en el modelo de tráfico macroscópico de flujo multimedia el cual refleja las relaciones entre las variables del tráfico como: la densidad de tráfico o la ocupación, velocidad y flujo. Diferenciándose del método convencional, las curvas son usadas para modelar el tráfico de flujo multimedia sin la suposición de ciertas formas de funciones. Además se tienen en cuenta la interrelación entre las variables.

Se pueden encontrar otros trabajos sobre el tráfico como (Kuang y Williamson, 2002; Chesire et al., 2001), donde se realizan estudios para el flujo multimedia de RealMedia y bajo el protocolo RTSP. En (Song, 2004) se estudia un algoritmo de control de tráfico de VoD usando metadatos para la renegociación del ancho de banda soportado por la red. En (Melendi et al., 2006) se presenta una aproximación para el despliegue de un servicio de vídeo en vivo basado en la tecnología de flujo multimedia sobre una red HFC. Además los autores consideran otros aspectos tales como, poner el servicio en operación tomando en cuenta nuevos aspectos como la orientación a mejorar y priorizar el análisis de servicios y presentan una arquitectura de servicios específicamente diseñada para redes HFC. En (García et al, 2007) se realiza una caracterización estadística del comportamiento de los usuarios así como de los flujos de tráfico intercambiados entre los clientes y el servidor utilizando tecnologías Real Networks. Se han tenido en cuenta aspectos como las características de las sesiones de usuario, reproducciones erróneas, volúmenes de tráfico de audio y vídeo transmitidos, tráfico de control, número y duración de las pausas de usuario, popularidad de los contenidos y perfiles de acceso diario por parte de los usuarios del servicio de VoD.

Con el objetivo de seguir mejorando la caracterización de los servicios de audio y vídeo reales desplegados, en (Melendi et al. 2009) y (García et al. 2009) se realizan nuevos estudios en los que se demuestra la existencia de dependencias estadísticas entre las diferentes interacciones de los usuarios con el sistema. Estos artículos no toman en cuenta la distribución a través de una red de cable para realizar los análisis. Aunque la mayor parte de los trabajos, entre ellos (Garcia et al. 2007) y (Griwod et al. 1997), que analizan el comportamiento de los usuarios consideran que el patrón de acceso sigue una distribución Zipf-Like, (Guo et al, 2007) han demostrado que en algunas ocasiones el acceso a los servicios multimedia puede modelarse utilizando una distribución del tipo exponencial extendida. Esta circunstancia puede emplearse para mejorar el modelado del usuario de los sistemas de VoD. Otro trabajo relacionado en el campo del modelado de servicios multimedia en Internet es el de (Tang et al. 2007). En él los autores proponen varios modelos de comportamiento del usuario y desarrollan un generador de carga sintético para realizar experimentos de emulación con este tipo de servicios. También relacionado con el modelado del usuario de servicios de VoD, (Lancieri y Duran, 
2006) estudian la redundancia parcial y global en los accesos de los clientes a los servicios multimedia en Internet. Su objetivo es descubrir la consistencia temporal en los accesos del usuario.

Este artículo presenta diferencias con los trabajos previos ya que ninguno de ellos toma en cuenta el análisis sobre uno de los protocolos más usados, el protocolo propietario de Adobe Systems RTMP, y a excepción de (Melendi et al., 2006), los trabajos previos no toman en cuenta la red HFC para su análisis.

\section{ENTORNO DE EXPERIMENTACIÓN}

Los experimentos se han realizado en el laboratorio de Televisión interactiva (iTV) de la Universidad de Oviedo en el que se ha construido un escenario de red de cable basado en el protocolo DOCSIS y se ha implementado un servicio de VoD utilizando tecnología Flash. Los componentes mínimos de infraestructura de red del laboratorio, se presentan en la Fig.1, así como su direccionamiento de red.

\section{Escenario de red}

Sistema de Terminación de Cable Módems (CMTS, por sus siglas en inglés): Es el dispositivo encargado de manejar la conexión a Internet a través de la red de cable. Realiza la codificación, modulación y gestión de acceso al medio compartido por los Módems de Cable (CM, por sus siglas en inglés). En general actúa como interfaz entre la red de datos y la red de Radio Frecuencia (RF). El CMTS usado es el Motorola BSR2000 basado en la DOCSIS 2.0 y el estándar PacketCable 1.0. Para el acceso a las redes provee dos interfaces Gigabit Ethernet y cuatro interfaces 10/100 BASE-T Ethernet, una interfaz 10/100 BASE-T para soporte de redundancia, un puerto T1/E1 y cuatro canales de subida y un canal de bajada para la conectividad DOCSIS (Motorola, 2005).

Módem de Cable: Es un tipo especial de módem diseñado para modular la señal de datos sobre una infraestructura de televisión por cable. Actúa como interfaz entre el computador personal (PC) y la red RF para distribuir el acceso a Internet de banda ancha, aprovechando el ancho de banda que no se utiliza en la red de cable. Los módems usados son Motorola SB510, los cuales, una vez encendidos, inician un intercambio de mensajes con el CMTS, que conducen al registro e inicialización permitiendo al CM estar listo para utilizar la red (CableLabs, 2001).

Servidor de Contenidos y Aplicaciones: Es un equipo PowerEdge 860 con un procesador a 2,4 GHz y sistema operativo Linux, Ubuntu, el cual se encarga del almacenamiento y adecuación de los contenidos y aplicaciones, para su posterior transporte por la red de cable. Se comunica con el CMTS a través de la interfaz de red Ethernet.

\section{Servidores Instalados}

Servidor de Flujo Multimedia: El servidor utilizado fue el FMS (Flash Media Server de Adobe Systems). Posee una arquitectura cliente servidor, El cliente es ActionScript y se ejecuta en Adobe Flash Player, Adobe AIR, o Adobe Flash Lite, mientras que el servidor es Server-Side ActionScript. El servidor y el cliente se comunican sobre una conexión persistente usando para ello el protocolo RTMP. Este protocolo utiliza TCP a nivel de la capa de trasporte y soporta el flujo multimedia codificado en formato FLV (Flash Video) ofrecida mediante el servidor FMS.

Cortafuegos: Se instaló la herramienta IPCop cuyo objetivo es servir de cortafuegos. Es una herramienta de código abierto y distribuida bajo términos de GNU. Básicamente proporciona una serie de servicios adicionales como DHCP (Dynamic Host Configuration Protocol) y puede establecer dos zonas bien diferenciadas: la zona verde ó red interna y la zona roja ó red exterior. 


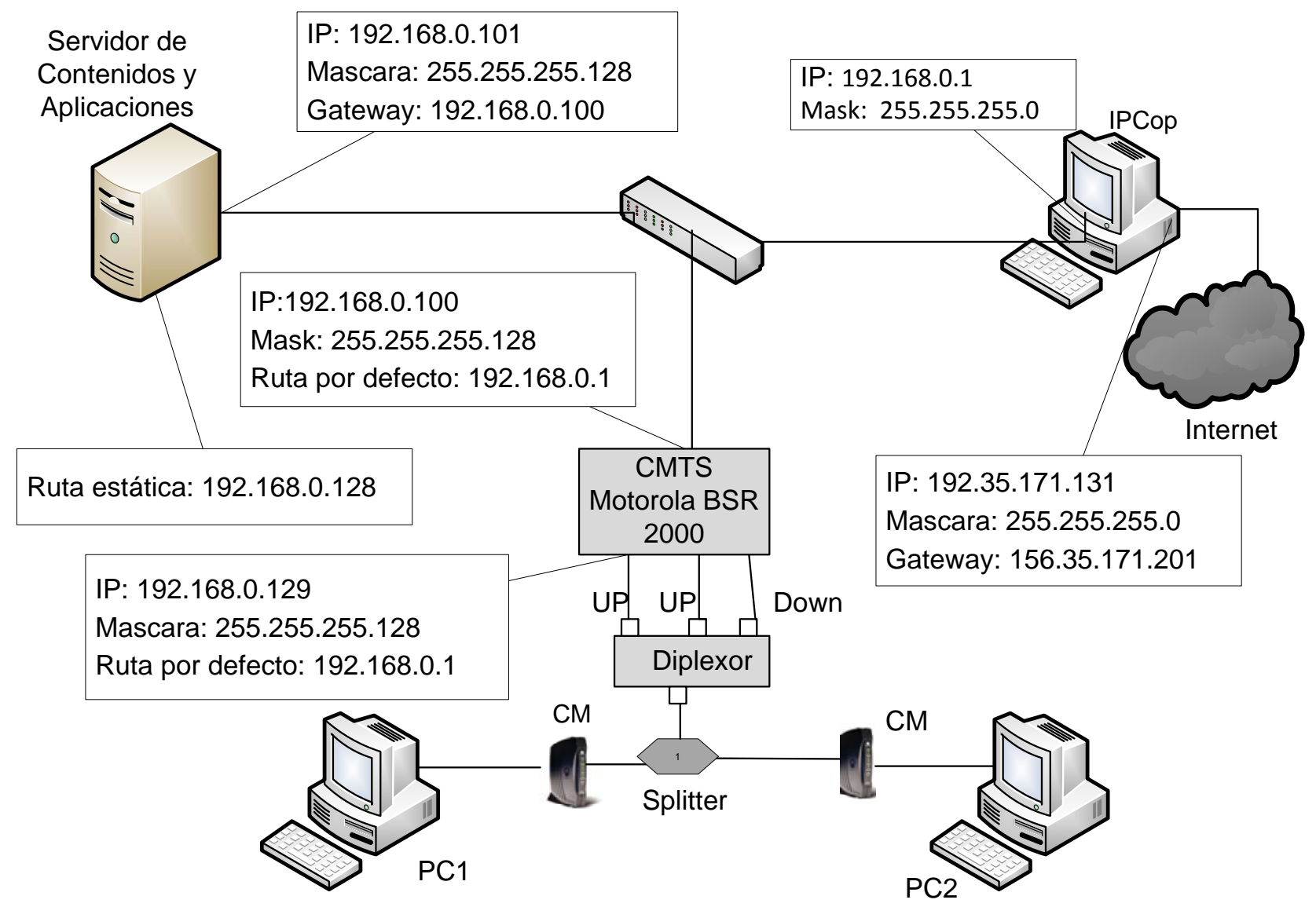

Fig. 1: Componentes y direccionamiento de la red HFC

Servidor de Direccionamiento: Se instaló un servidor DHCP en el servidor de contenidos y aplicaciones. Es el encargado de proporcionar una dirección a los CM para que puedan establecer una comunicación IP, además de entregar el nombre del archivo de configuración que debe aceptar el CM. Ya que las redes de cable y la Ethernet pertenecen a rangos distintos, es necesario que el servidor DHCP tenga una máscara de red que contenga a las dos subredes.

Servidor TFTP (Trivial File Transfer Protocol): Instalado en el Servidor de Contenidos y Aplicaciones, es el encargado de transferir el archivo de configuración del CM.

Como paso final a la configuración necesaria para el correcto funcionamiento de la red se crea en el servidor de contenidos y aplicaciones, una ruta estática hacia la red de cable, a través de la interfaz Ethernet del CMTS.

\section{ANÁLISIS DE TRÁFICO}

Las medidas del tráfico generado por el servidor de aplicaciones a solicitud del servicio de VoD desde los PC1 y PC2 son tomadas mediante el analizador de protocolos Wireshark, ver Fig.1.

\section{Medidas de tráfico sobre el servidor FMS}

Se tomaron medidas de tráfico con diferentes vídeos, utilizando como parámetros de codificación los siguientes: Audio a $32 \mathrm{Kbps}$ y $64 \mathrm{Kbps}$ y Vídeo a 144, 528 y $1008 \mathrm{Kbps}$. Observando el comportamiento del tráfico en volumen de bytes transferidos por segundo. En la Fig. 2, se ve una conducta similar para todas las calidades, presentándose ciertas diferencias para las calidades de vídeo de 1008 Kbps. 
En la Fig. 2, las curvas superiores en cada recuadro representan el tráfico total y las curvas inferiores representan el tráfico generado por el protocolo RTMP (medido en Kbytes/s) para las diferentes calidades de flujo multimedia. Dicha información está representada en forma de series a través del tiempo. Esta información, tabulada en este formato, no es de utilidad cuando se trata de obtener una conducta basada en variabilidad con cierto comportamiento probabilístico. Por esta razón para cada una de las curvas inferiores de la Fig. 2. se realiza la prueba de bondad de ajuste de KolmogorovSmirnov (Astaiza et al., 2007), que permite obtener las distribuciones estadísticas para cada una de las curvas de tráfico capturadas.
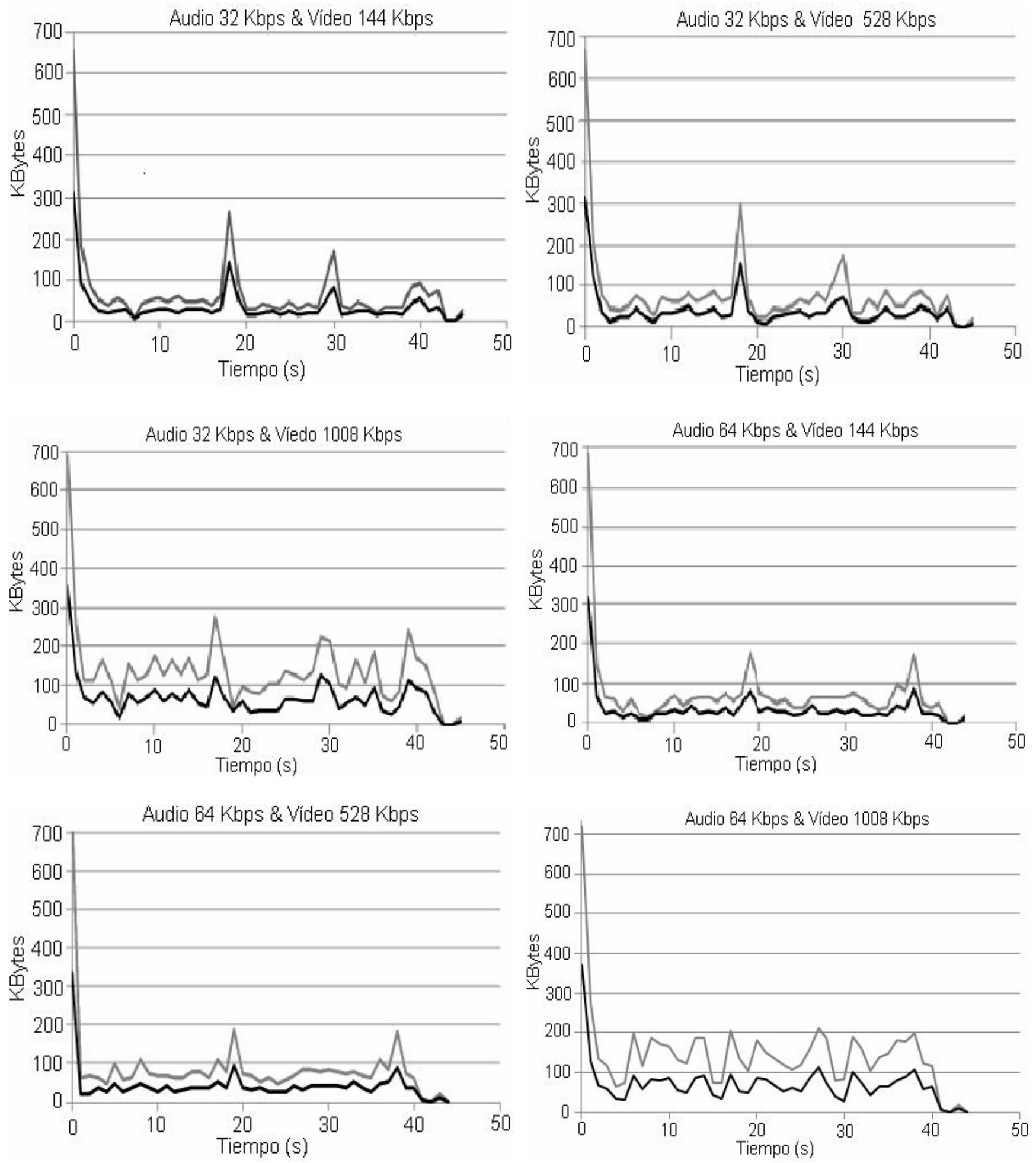

Fig. 2: Capturas de tráfico para diferentes calidades de flujo multimedia 
La Fig.3, presenta el histograma para una de las muestras. Se observa que el tamaño de los paquetes es 1514 Bytes en más del $97 \%$ de los casos. Por ello, la mejor distribución estadística para caracterizar el tamaño de estos paquetes es una distribución constante de 1514 Bytes. Se debe tener en cuenta que éste es el tamaño de la trama Ethernet, por lo que es necesario restarle las 14 Bytes de la cabeceras Ethernet, 20 Bytes de la cabecera IP, y 20 Bytes de la cabecera TCP para obtener el tamaño del paquete RTMP. El comportamiento es el mismo para las otras muestras.

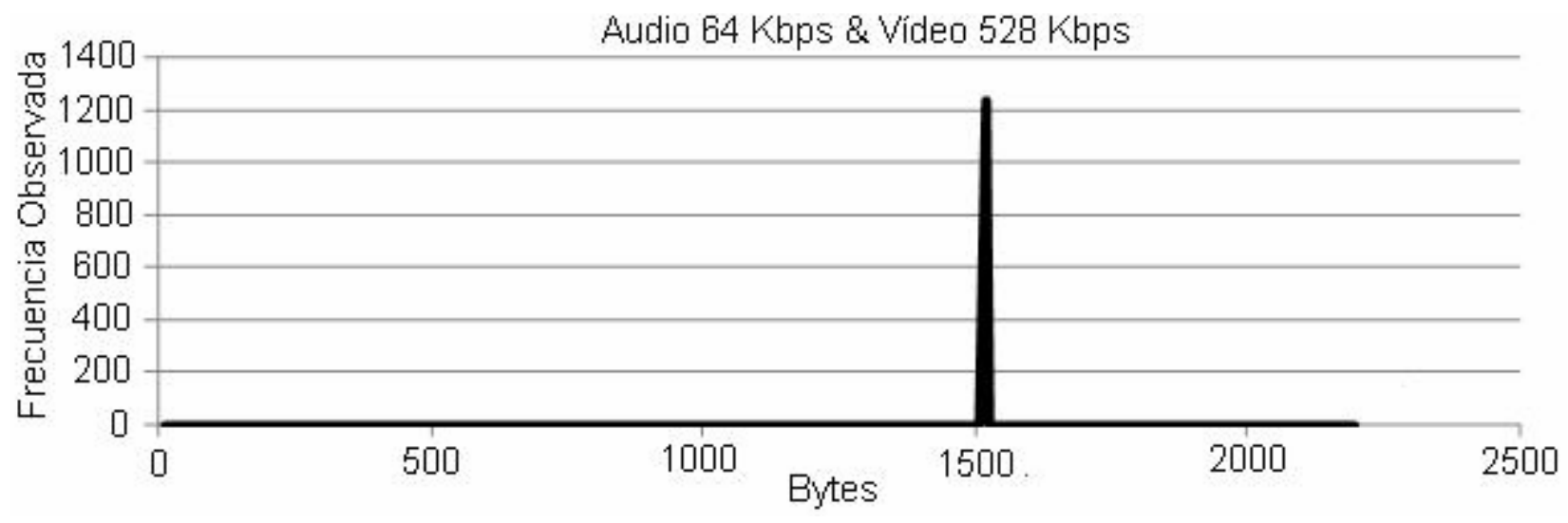

Fig. 3: Tamaño de los paquetes

En lo que respecta al análisis temporal de los datos, se observa que existen muchos valores pequeños de tiempos entre paquetes, de forma casi periódica. Esto se debe a que el tráfico tiene un comportamiento a ráfagas debido al proceso de codificación y envío de tramas de diferente tamaño. En la Fig. 4, se verifica este efecto, donde se muestra la curva para una de las muestras, del número de paquetes en función del tiempo.

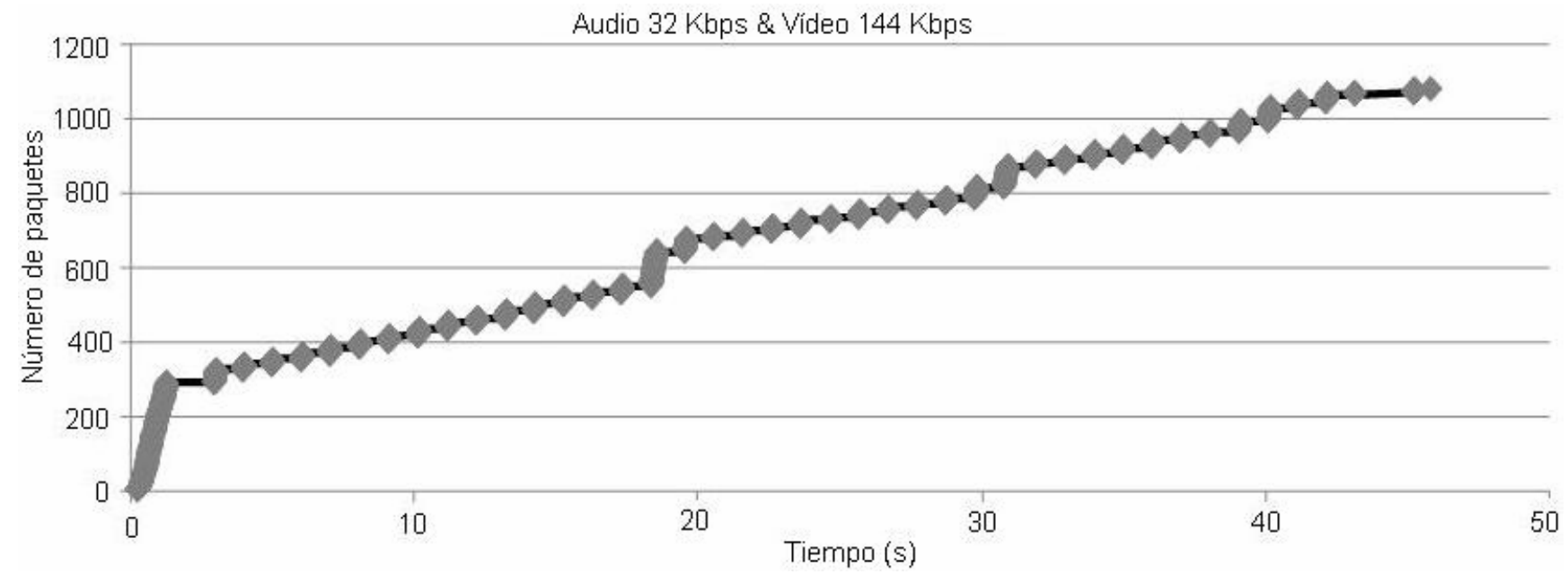

Fig. 4: Comportamiento a ráfagas del tráfico

Teniendo en cuenta este comportamiento del tráfico, es necesario para su caracterización obtener las Funciones de Distribución de Probabilidad (FDP) para el tamaño de las ráfagas en número de paquetes y el tiempo entre ráfagas.

\section{FDP para el tamaño de las ráfagas}

Para validar las distribuciones estadísticas que modelan el comportamiento real, se ha utilizado las pruebas de bondad de ajuste de Kolmogorov-Smirnov (K-S). Para ello, se obtiene el valor estadístico Dn global de K-S, el cual calcula la distancia máxima entre la distribución acumulada de la muestra $\mathrm{Fn}(\mathrm{x})$ y la FDP que se ajusta al comportamiento de la muestra $\mathrm{F}(\mathrm{x})$, ver ecuación 1 (Astaiza et al., 2007). 
El parámetro Dn, corresponde al menor valor arrojado por el paquete STATGRAPHICS Plus 5.1, para cada FDP que valida la hipótesis de que las muestras procedan de sus correspondientes distribuciones con un nivel de confianza de al menos un $90 \%$.

En la tabla 1, se muestran los resultados de las pruebas de bondad de ajuste para cada uno de los flujos multimedia (Uno a Seis), la FDP, sus parámetros y el valor estadístico Dn global de K-S (Law y Kelton, 2000).

Tabla 1: Resultados de las pruebas de K-S para el tamaño de las ráfagas

\begin{tabular}{|l|c|c|l|l|c|}
\hline $\begin{array}{l}\text { Difusión } \\
\text { multimedia }\end{array}$ & $\begin{array}{l}\text { Audio } \\
\text { Kbps }\end{array}$ & $\begin{array}{l}\text { Vídeo } \\
\text { Kbps }\end{array}$ & \multicolumn{1}{|c|}{ FDP } & \multicolumn{1}{|c|}{ Parámetros } & Dn \\
\hline Uno & 32 & 144 & Laplace & $\begin{array}{l}\text { Escala }=0,17 \\
\text { Media = 15 }\end{array}$ & 0,16 \\
\hline Dos & 32 & 528 & Logística & $\begin{array}{l}\text { Desviación Típica }=11,16 \\
\text { Media = 23,44 }\end{array}$ & 0,08 \\
\hline Tres & 32 & 1008 & Gamma & $\begin{array}{l}\text { Escala }=0,15 \\
\text { Forma }=7,28\end{array}$ & 0,09 \\
\hline Cuatro & 64 & 144 & Laplace & $\begin{array}{l}\text { Escala }=0,15 \\
\text { Media = 18 }\end{array}$ & 0,16 \\
\hline Cinco & 64 & 528 & Chi-Cuadrado & Grados de libertad = 25,78 & 0,10 \\
\hline Seis & 64 & 1008 & Normal & $\begin{array}{l}\text { Desviación Típica }=15,03 \\
\text { Media = 46,28 }\end{array}$ & 0,09 \\
\hline
\end{tabular}

El análisis muestra diferentes FDP según las calidades de codificación, lo cual puede dificultar la generación de un modelo para su simulación. Sin embargo durante el proceso de las pruebas de bondad de ajuste se observa que todas las muestras pueden ser caracterizadas mediante una FDP de Laplace cuyos parámetros se muestran en la tabla 2.

Tabla 2: Parámetros para la FDP de Laplace para los diferentes flujos multimedia

\begin{tabular}{|l|c|c|c|c|c|c|}
\cline { 2 - 7 } \multicolumn{1}{c|}{} & Uno & Dos & Tres & Cuatro & Cinco & Seis \\
\hline Media & 15 & 24,5 & 43,0 & 18 & 24,5 & 46,5 \\
\hline Escala & 0,17 & 0,11 & 0,08 & 0,15 & 0,15 & 0,08 \\
\hline Dn & 0,16 & 0,15 & 0,13 & 0,16 & 0,12 & 0,13 \\
\hline
\end{tabular}

FDP para el tiempo entre ráfagas:

Se tienen en cuenta los mismos conceptos respecto al parámetro Dn del punto anterior. Durante el proceso de las pruebas de bondad de ajuste se observa que todas las muestras pueden ser caracterizadas mediante una distribución Normal, cuyos parámetros se muestran en la tabla 3.

Tabla 3: Parámetros para la FDP Normal para los diferentes flujos multimedia

\begin{tabular}{|l|c|c|c|c|c|c|}
\cline { 2 - 7 } \multicolumn{1}{c|}{} & Uno & Dos & Tres & Cuatro & Cinco & Seis \\
\hline Media & 0,97 & 0,95 & 0,92 & 0,96 & 0,94 & 0,93 \\
\hline $\begin{array}{l}\text { Desviación } \\
\text { Típica }\end{array}$ & 0,02 & 0,02 & 0,03 & 0,02 & 0,02 & 0,02 \\
\hline Dn & 0,081 & 0,11 & 0,11 & 0,07 & 0,08 & 0,10 \\
\hline
\end{tabular}

Queda así caracterizado el comportamiento del protocolo RTMP mediante la identificación de las funciones de distribución estadística para el tamaño de los paquetes; el tiempo entre ráfagas y los tamaños de las mismas medidas en número de paquetes. 


\section{MODELO DEL SERVICIO DE FLUJO MULTIMEDIA}

Una vez caracterizado el servicio de flujo multimedia, los parámetros identificados para cada una de las funciones deben ser los parámetros de entrada a las herramientas de simulación. El modelo es construido mediante la herramienta de simulación OPNET Modeler. La herramienta permite diferentes escenarios de simulación con varios usuarios y perfiles, en conjunto con otras aplicaciones para determinar las capacidades de la red de acceso HFC para proporcionar una adecuada prestación de un servicio de VoD.

\section{Modelo del Cliente y del Servidor}

En la simulación de servicios de flujo multimedia, los clientes corresponden a estaciones de trabajo que se conectan a través de una interfaz Ethernet al CM. En estas estaciones se configuran los perfiles que representan el comportamiento de un usuario que interactúa con las aplicaciones de VoD. El servidor se configura de tal forma que pueda enviar los archivos y responder a las peticiones realizadas por los diferentes usuarios.

\section{Configuración DOCSIS}

En la tabla 4, se muestran los parámetros de configuración del protocolo DOCSIS tenidos en cuenta durante esta investigación.

Tabla 4. Configuración del protocolo DOCSIS

\begin{tabular}{|c|c|c|}
\hline Configuración DOCSIS & Canal Descendente & Canal Ascendente \\
\hline Modulación & $256 \mathrm{QAM}$ & QPSK \\
\hline Tasa de datos & $51 \mathrm{Mbps}$ & $2.56 \mathrm{Mbps}$ \\
\hline Ancho de banda del canal & $8 \mathrm{MHz}$ & $800 \mathrm{KHz}$ \\
\hline Frecuencia central & $550 \mathrm{MHz}$ & $12 \mathrm{MHz}$ \\
\hline Versión DOCSIS & \multicolumn{2}{|c|}{1.1} \\
\hline Opción de Fragmentación & \multicolumn{2}{|c|}{ Habilitada } \\
\hline
\end{tabular}

\section{RESULTADOS}

\section{Escenario de pruebas uno}

Involucra seis clientes, que corresponden a las dos calidades de audio y a las tres calidades de vídeo cuyo modelo matemático fue encontrado en la sección Modelo de Tráfico, donde dichos clientes hacen solicitudes del servicio de flujo multimedia de manera simultánea. Los resultados fueron los siguientes:

Retardo DOCSIS: EI Retardo DOCSIS se mide a partir del momento en que se encola una trama para su transmisión en la capa MAC DOCSIS en el lado del transmisor (ya sea CM o CMTS) hasta el momento en que se entrega al receptor. Es importante tenerlo en cuenta, ya que influye notablemente en el retardo final del tráfico de la aplicación multimedia. Su valor sobre la red empieza a incrementarse con el tiempo a medida que se ejecutan todos los flujos multimedia de manera simultánea sobre los clientes, hasta estabilizarse en un valor de retardo DOCSIS de 19 ms a los 40 segundos de iniciarse las transmisiones.

Retardo de las Aplicaciones: representa el tiempo que tardan en llegar a su destino los paquetes de la aplicación de VoD. El flujo multimedia Seis, por poseer los mayores valores de codificación, presenta el mayor retardo $(95 \mathrm{~ms})$ de las calidades evaluadas. El flujo multimedia Uno, con los menores valores de codificación, presenta el menor retardo $(25 \mathrm{~ms})$. El valor promedio del retardo de las seis aplicaciones es de $60 \mathrm{~ms}$. Con estos valores se puede concluir que la red HFC es capaz de soportar los flujos multimedia, dentro del límite de 200 ms establecido en ITU-T_G.1010, 2001. 
Velocidad efectiva de transferencia de datos: en el canal descendente es mucho mayor, alcanzando un valor máximo de $1700 \mathrm{Kbps}$, el cual hace referencia a todos los flujos multimedia enviados a cada uno de los clientes. En el canal ascendente alcanza un valor máximo de $135 \mathrm{Kbps}$, el cual corresponde a los mensajes de confirmación (ACK)

\section{Escenario de pruebas dos}

Para tener una mayor exigencia de la red se configuraron catorce flujos multimedia de la siguiente forma: Tres clientes ejecutando dos flujos multimedia cada uno, que corresponden a los flujos multimedia Dos y Tres; dos clientes ejecutando cuatro flujos multimedia cada uno, que corresponden a los flujos multimedia Uno, Cuatro, Cinco y Seis.

Retardo DOCSIS: presenta un comportamiento similar al descrito en el escenario de prueba uno, donde el retardo empieza a incrementarse a medida que transcurre de manera simultánea la ejecución de los catorce flujos multimedia sobre los clientes, hasta estabilizarse en un valor de retardo DOCSIS de $50 \mathrm{~ms}$ a los $40 \mathrm{~s}$ de iniciarse las transmisiones.

Retardo de las Aplicaciones: el valor promedio de este retardo alcanza un valor de $180 \mathrm{~ms}$, manteniéndose dentro del límite de 200 ms establecido en ITU-T_G.1010, 2001.

Velocidad efectiva de transferencia de datos: en el canal descendente alcanza valores pico de 2,5 Mbps, el cual hace referencia a todos los flujos multimedia enviados a cada uno de los clientes. La velocidad efectiva de transferencia de datos del canal ascendente alcanza un valor máximo de 200 Kbps.

Con un retardo de $180 \mathrm{~ms}$ los escenarios de prueba se están acercando al límite máximo permitido de los 200 ms, por lo que se pueden seguir aumentando el número de usuarios hasta encontrar un número de usuarios que desborden el límite de retardo máximo de $200 \mathrm{~ms}$, sin embargo esto dependerá además de las características de codificación de las aplicaciones.

\section{CONCLUSIONES}

Este artículo provee un modelo matemático que describe el comportamiento de un servicio de VoD, basado en el protocolo RTMP sobre una red HFC, el cual presenta como característica un comportamiento a ráfagas, para las diferentes calidades de codificación. Este modelo ha sido construido basándose en las capturas de tráfico reales sobre la red de cable del laboratorio de iTV de la Universidad de Oviedo. A partir de este modelo se puede deducir el comportamiento de la red ante el aumento de usuarios o manejo de diferentes aplicaciones.

Con el soporte de la herramienta OPNET Modeler, se simuló el modelo del servicio de VoD permitiendo la captura de las curvas de tráfico y el respectivo análisis de los parámetros de rendimiento, como el retardo DOCSIS y la velocidad efectiva de transferencia de datos.

Los resultados de la simulación muestran que la velocidad efectiva de transferencia de datos es similar entre el servidor de aplicaciones y el CMTS durante las primeras decenas de segundos, después de las cuales la velocidad efectiva de transferencia de datos del canal descendente es mucho mayor que el del canal ascendente. Este resultado está acorde con lo esperado, ya que es el Servidor de Aplicaciones quien entrega volúmenes mayores de información correspondiente a las ráfagas del servicio de flujo multimedia hacia la red, mientras que el tráfico de subida está conformado por las solicitudes de los diferentes clientes, los paquetes de establecimiento de la conexión y los paquetes de confirmación de recepción de la información.

El modelo permite que se cree la base del conocimiento y hacer proyecciones que pueden servir para la toma de decisiones sobre el uso del protocolo RTMP, para los proveedores de contenidos que deseen ampliar su oferta de servicios sobre sus redes. 
La posibilidad de trabajar en entornos de emulación, donde el servicio multimedia es real y la parte de red es simulada permite evaluar el tráfico real del servicio sin requerir de una infraestructura real de red. Así, se podrán construir generadores de carga que simulen el comportamiento de un número elevado de clientes, accediendo a través de la red simulada a un servidor real de vídeo. En este escenario es posible validar el rendimiento real del servicio en diferentes entornos de red y compitiendo tanto con cargas de tráfico simulado como con otros tipos de tráfico real procedentes de otras aplicaciones.

El objetivo final de este trabajo es construir modelos de simulación más realistas. Así, se evaluará escenarios alternativos para la distribución de tráfico multimedia en las redes de comunicaciones. Por ejemplo, se debe estudiar la configuración de los dispositivos de la red de cable para lograr una mejora en su funcionamiento, tanto del servicio de flujo multimedia como del resto de servicios. Se podrá evaluar el rendimiento ante diferentes configuraciones del servicio en conjunto con otras aplicaciones, para determinar las capacidades de la red de acceso HFC y su protocolo DOCSIS. También es posible la realización de estudios de tráfico teniendo en cuenta condiciones de interactividad en una red HFC y por su puesto en otros contextos como puede ser investigaciones que involucren el canal de retorno de la red HFC, para descargar tráfico de flujo multimedia bajo RTMP.

\section{AGRADECIMIENTOS}

Este trabajo es apoyado por el proyecto ST-CAV, código 1103-489-25425, financiado por el COLCIENCIAS y el SENA; el proyecto MT2TDi, ID 2590, financiado por la Vicerrectoría de Investigaciones de la Universidad del Cauca y el proyecto SOLITE, financiado por CYTED.

\section{REFERENCIAS}

Álvarez-Campana M., Berrocal J., González F., Pérez R., Román I. y Vázquez E, Tecnologías de Banda Ancha y Convergencia de Redes. Ministerio de Industria, Turismo y Comercio, Madrid, España (2009).

Astaiza, E., Bermudez, H. y Muñoz. P, Simulación de sistemas de telecomunicaciones, 45-56, Arte Imagen, Armenia, Colombia (2007).

CableLabs, Data over Cable System Interface Specifications Radio frequency interface Specification (2001).

CableLabs, Radio Frequency Interface Specification DOCSIS 2.0, Abril (2006).

Chen, D., Zhang, J., Wang, J. y Wang, F-Y, Freeway traffic stream modeling based on principal curves, Intelligent Transportation Systems. IEEE: 1, 368-372 (2003).

Chesire, M., Wolman, A., Voelker. y G, Levy, Measurement and analysis of a streaming-media workload, Proceedings of the USENIX Symposium on Internet Technologies and Systems (2001).

España, M, Servicios Avanzados de Telecomunicación, Díaz Santos, Madrid, España (2003).

ETSI EN 300744 V1.5.1, Digital Video Broadcasting (DVB); Framing structure, channel coding and modulation for digital terrestrial television, EBU-UER, Geneva, Switzerland (2004).

García, R. G., Pañeda, X., Melendi, D. y García, V, Probabilistic analysis and interdependence discovery in the user behaviour of a video news on demand service. In Elsevier Computer Networks, Volume 53, Issue 12, p.p. 2038-2049, Agosto (2009).

García, R., Pañeda, X., García, V., Melendi, D. y Vilas, M, Statistical characterization of a real video on demand service: User behaviour and streaming-media workload analysis, Simulation Modelling Practice and Theory, 15(3), 672-689 (2007). 
Guo, L., Tan, T., Chen, S., Xiao, Z. y Zhang, X, Does Internet Media Traffic Really Follow Zipf-like Distribution?, in ACM-SIGMETRICS, Junio (2007).

Kuang, T. y Williamson C, A measurement study of RealMedia streaming traffic, Proceedings of ITCOM (2002).

Lancieri, L. y Durand, N, Internet user behaviour: compared study of the access traces and applications to the discovery of communities, in IEEE Transactions on Systems, Man and Cybernetics-Part A: Systems and Humans, vol. 36, no. 1, Enero (2006).

Law, A. y Kelton, W, Simulation Modelling and Analysis. McGraw-Hill International Series (2000).

Loguinov, D. y Radha, H, Measurement study of low-bitrate internet video streaming, Internet Measurement Conference archive. Proceedings of the 1st ACM SIGCOMM Workshop on Internet Measurement, 281-293, San Francisco, California, USA (2001).

Melendi, D., García, R. G., Pañeda, X. y García, V, Multivariate distributions for workload generation in video on demand systems. In IEEE Communication Letters, Volume 13, Issue 5, p.p. 348-350, Mayo (2009).

Melendi, D., Pañeda, X., García, R., Bonis, R. y García. R, Deployment of Live-Video Services Based on Streaming Technology Over An HFC Network, e-Business and Telecomunication Networks, Springer, pp 256-263, Netherlands (2006).

Melendi, D., Pañeda, X., García, V., García, R. y Neira, A, Métricas para el Análisis de Calidad en Servicios de Vídeo-Bajo-Demanda Reales, III congreso Iberoamericano de Telemática (CITA), Montevideo Uruguay, 30 y 31 de Octubre (2003).

Mena, A. y Heidemann J, An Empirical Study of Real Audio Traffic, INFOCOM, Nineteenth Annual Joint Conference of the IEEE Computer and Communications Societies. IEEE: 1, 101-110 (2000).

Merwe, J., Sen, S. y Kalmanek, C, Streaming Video Traffic: Characterization and Network Impact, In Proceedings of International Workshop on Web Content Caching and Distribution, Boulder, Colorado, USA (2002).

Motorola, BSR 2000 Installation Guide, Release 1.0 (2005).

Song, $\mathrm{H}$, Metadata-Based Video-on-Demand Traffic Control over the Network Supporting Bandwidth Renegotiations, IEICE Trans Commun, E87-B(5),1373-1381 (2004).

Tang, W., Fu, Y., Cherkasova, L. y Vahdat, A. Modeling and generating realistic streaming media server workloads. in Computer Networks, 51 (1), 336-356 (2007). 